\title{
Représentations et extensions d'algèbres de Jordan
}

\author{
J. Ravatin et H. ImmÉdiato \\ Département de Mathématiques - Faculté des Sciences de Lyon, France
}

Received May 25; in revised form November 5, 1969

\begin{abstract}
This article is a study on polynomial algebras defined from Jordan algebras. The consideration of $*$ continuous representations of a Jordan algebra leads to a general theorem for the decomposition of cyclic representations.
\end{abstract}

\section{Introduction}

De récents articles de P. Jordan [1] et Ion [2] font ressortir l'intérêt des algèbres non-associatives en Physique quantique. Comme le fait remarquer P. Jordan une analyse mathématique de la mécanique quantique peut poursuivre deux buts différents; soit une approche logique [3], soit une extension de la théorie quantique (1). Quelque soient ces buts, la théorie des $*$ représentations continues s'avérait nécessaire [4] et [5]. D'autre part les algèbres de polynômes étant utiles dans le cas associatif pour les Physiciens, nous avons étendu au cas non-associatif les résultats de celles-ci [8]. On voit aisément que les résultats obtenus sur ces algèbres de polynômes ne sont pas spécifiques aux algèbres de Jordan, en cela, nous ne suivons pas la voie de Braun et Koecher [6] mais étendons les définitions de Bourbaki [7]. Celles-ci pourraient tout aussi bien être appliquées à toute algèbre non-associative, pourvu qu'elles soient de puissance associative. On ne peut donc appeler ces polynômes «Polynômes de Jordan».

.Par contre, à chaque fois que cela est possible, les propriètés sont étudiées sur des corps plus généraux que le corps des complexes 'caractéristiques différentes de 2 et 3) qui est choisi très souvent en Physique Quantique.

Le détail des propositions, signalées dans les rappels peut être trouvé dans [8], ainsi que certaines démonstrations de propriétés se rapportant aux représentations.

Les résultats relatifs aux représentations unitaires continues prolongent l'étude entreprise dans [4] et [5]. 


\section{Definitions}

Soit une algèbre de Jordan $A$, commutative, avec unité, sur un corps $K$, de caractéristique différente de 2 et 3 .

Soir $B$ une algèbre de Jordan sur $K$ telle que $A$ soit une sous-algèbre de Jordan unitaire de $B$. On appelle polynôme à coefficients dans $A$, ou polynôme sur $A$, toute suite finie $R_{n}$ d'applications de $B$ dans $B$ construites de la manière suivante:

(i) Les $R_{0}$ sont des applications constantes de $B$ dans $A$.

(ii) Les $R_{1}$ sont des applications linéaires, éléments de la sousalgèbre associative, engendrée par les éléments de $A$ dans l'algèbre multiplicative de $B$.

(iii) Les $R_{n}$ sont construits comme sommes finies de produits finis:

et

$$
R_{n_{1}} \ldots R_{n_{j}} \text { avec } n_{1}+\cdots+n_{j}=n
$$

$$
R_{n} R_{n^{\prime}}(x)=R_{n}(x) R_{n^{\prime}}(x)
$$

et avec comme conditions supplémentaire $R_{n}(x)=R_{n}(x)$.

\section{Rappels [8]}

L'ensemble des polynômes sur $A$ est une algèbre de Jordan $\mathscr{P}(A)$. On peut identifier $A$ à une sous-algèbre de $\mathscr{P}(A)$.

Soit $X$ le polynôme: $P_{0}=0$,

$$
\begin{aligned}
& P_{1}=I_{B} \text { (identité de } B \text { ), } \\
& P_{n}=0, \forall n \geqq 2 .
\end{aligned}
$$

Alors tout polynôme $f$ sur $A$ peut s'écrire:

$$
f=a_{0}+P_{1}(X)+\cdots+P_{n}(X) .
$$

L'algèbre de Jordan des polynômes $f$ sur $A$ est notée $A[X]$. Pour $x$ fixé, considérons l'application $\theta_{x}: f \rightarrow f(x)$ de $A[X]$ dans $B$. Alors $A$ est une sous-algèbre de Jordan de l'algèbre de Jordan $\theta_{x}(A[X])$ et cette dernière est une sous-algèbre de Jordan de $B$. On pose $\theta_{x}(A[X])=A[x]$.

On appelle algèbre de Jordan polynomiale sur une algèbre de Jordan $A$, toute algèbre de Jordan $A^{\prime}$ vérifiant les axiomes suivants:

(i) $A$ est une sous-algèbre unitaire de $A^{\prime}$.

(ii) $A^{\prime}$ contient au moins un élément $x$ transcendant.

(iii) $A^{\prime}=A[x]$.

\section{Ideal engendré par un élément dans une algèbre de Jordan}

1. Proposition 1. Soit $A$ une algèbre de Jordan, $x \in A$. Alors l'idéal engendré par $x$ est l'image par $\theta_{x}: f \rightarrow f(x)$ de l'ensemble des polynômes sur A sans terme constant. 
En effet si $f(x)$ est l'image par $\theta_{x}$ d'un polynôme $f$ à terme constant nul, alors pour tout $x \in A, a f(x)=(a f)(x)$, et comme $f$ n'a pas de terme constant il en est même pour af donc $a f(x)$ est l'image par $\theta_{x}$ d'un polynôme sans terme constant; or ceci suffit à montrer que l'ensemble des $f(x)$ avec $f(x)=0$ est un idéal; la suite de la démonstration étant évidente. D'autre part $f \in A[X]$ ce qui entraine que $f(x)$ appartient à l'idéal engendrépar $x$, ce qui démontre la proposition.

2. Extensions d'algébres de Jordan. Soit $A$ une algèbre de Jordan commutative, unitaire, sur $K$. On appelle extension de $A$, toute algèbre de Jordan $B$ sur $K$ telle que $A$ soit une sous-algèbre unitaire de $B$.

Soit $B$ une extension de $A$; alors $x$ appartient à $B$ si $x$ est algèbrique sur $A$. Soit $I$ l'ensemble des $f$ appartenant à $A[X]$ tels que $f(x)=0$; on voit aisément que $I$ est un idéal de $A[X]$. Pour le cas des extensions algèbriques ou transcendantes on consultera [8].

3. Extensions simples. a) Définition. Soit $A$ une algèbre de Jordan, $B$ une extension de $A, x$ qui qui appartient à $B$. Considérons la sousalgèbre de $B$, engendrée par $A \cup\{x\}$. On l'appelle l'extension simple de $A$ dans $B$ par adjonction de l'élément $x$.

b) Proposition 2. La sous-algèbre de B engendrée par $A \cup\{x\}$ est égale à $A[x]$ :

Soit $A_{x}$ la sous-algèbre engendrée par $A \cup\{x\}$. Or $A \subseteq A[x]$ et $x \in A[x]$ donc $A_{x} \subseteq A[x]$. D'autre part si $f_{n}(x)$ est un monôme de degré $n$, $f_{n}(x) \in A_{x}$ donc pour tout $f \in A[X], f(x) \in A(x)$, donc $A[x] \subseteq A_{x}$ et on a l'égalité cherchée.

Si $x$ est algébrique sur $B$, on va considérer $I$ idéal dans

$$
\{f \mid f \in A[X] \text { tel que } f(x)=0\} .
$$

$A[X] / I$ est aussi une algèbre de Jordan; à $\bar{f}$ qui appartient à cette dernière algèbre de Jordan on peut faire correspondre $f(x)$.

Soit $\bar{\theta}: \bar{f} \rightarrow f(x) . \bar{\theta}_{x}$ est surjective de $A[X] / I$ dans $A[x]$ car $A[x]$ $=\theta_{x_{x}}(A[X])$.

$\bar{\theta}_{x}$ est injective car $f(x)=0$ entraine $f \in I$ donc $\bar{f}=0$. On vérifie aisément que $\theta_{x}$ est un homomorphisme d'algèbre donc $\bar{\theta}_{x}$ est un isomorphisme de $A[X] / I$ sur $A[x]$.

\section{Introduction d'une topologie}

1. Généralités. Soit $B$ une algèbre de Jordan normée par une norme telle que $\|x y\| \leqq\|x\|\|y\|, A$ une sous-algèbre unitaire de $B$.

Soit $f_{p}$ un monôme de degré $p$. On pose $\left\|f_{p}\right\|=\sup \left\|f_{p}(x)\right\|$. Pour une forme $R_{p}=\sum_{p=0}^{n} f_{p}$, de degré $p(8)$, on pose:

$$
\left\|R_{p}\right\|=\sup _{x}\left\|R_{p}(x)\right\| .
$$


Pour un polynôme $f_{n}=\sum_{p=0}^{n} R_{p}(X)$, on pose $\left\|f_{n}\right\|=\sum_{p=0}^{n}\left\|R_{p}\right\|$. Pour une série on se repportera à [8].

On appelle série convergente une série dont la norme est finie et on voit que l'ensemble des séries est une algèbre normée [8].

2. Étude de l'algèbre des polynômes sur $A$ en tant qu'algèbre normée. Soit une série convergente: $\left(f_{p}\right)$. Considérons les $f_{p}(x)$ pour $\|x\| \leqq 1$

$$
\left\|\sum_{p=0}^{k} f_{p}(x)\right\| \leqq \sum_{p=0}^{k}\left\|f_{p}(x)\right\| .
$$

Alors

$$
\begin{gathered}
\lim _{k \rightarrow \infty}\left\|\sum_{p=0}^{k} f_{p}(x)\right\| \leqq \lim _{k \rightarrow \infty} \sum\left\|f_{p}(x)\right\| \leqq\|\sigma\| \\
\cdot\left\|\sigma-\sum_{p=0}^{k} f_{p}(x)\right\|=\lim _{p \rightarrow \infty} \sum_{k=0}^{k}\left\|f_{k+n}\right\| \leqq\|\sigma\|-\sum_{p=0}^{k}\left\|f_{p}\right\| .
\end{gathered}
$$

Si $k \rightarrow \infty$, alors $\sum_{p=0}^{k}\left\|f_{p}\right\| \rightarrow\|\sigma\|$ donc $\lim _{k \rightarrow \infty} \lim _{p \rightarrow \infty} \sum_{n=0}^{p}\left\|f_{k+n}\right\|=0$ donc $\lim _{k \rightarrow \infty}\left\|f_{k}\right\|=0$ et $\left\|\sigma-\sum_{p=0}^{k} f_{p}(x)\right\|$ tend vers zéro.

Soit $x$ tel que $\|x\| \leqq 1$. Alors $\sum_{p=0}^{k} f_{p}(x)$ est borné et la suite $\left(\left\|\sum_{p=0}^{k} f_{p}(x)\right\|\right)$ est une suite de Cauchy dans $R$ et il existe alors $\lim _{m}\left\|\sum_{p=0}^{m} f_{p}(x)\right\|$.

Considérons le complété $\bar{B}$ de $B$. Alors la suite $\left(\sum_{p=0}^{n} f_{n}(x)\right)$ est une suite de Cauchy dans $B$. Elle converge donc dans $\bar{B}$. Soit $\sigma(x)$ sa limite, on a donc $\sigma(x)=\sum_{p=0}^{\infty} f_{p}(x)$.

Alors toutes les séries convergentes dans $B$ sont uniformément convergentes dans la boule unité de $B$ et dans celle de $\bar{B}$.

On a ainsi un théorème de Cauchy dans $\bar{B}$ pour les séries à coefficient dans $A$.

3. Conséquences. Si on considère $\overline{A[x]}$, on a $\overline{A[x]}=A[[x]]$ pour $\|x\| \leqq 1$, où $A[[x]]$ est l'image de l'ensemble des séries sur $A$ par l'homomorphisme $\sigma \rightarrow \sigma(x)$.

Soit $B$ une algèbre de Jordan normée complète, $A$ une sous-algèbre unitaire normée complète. $A$ en tant que sous-espace fermé admet un supplémentaire, notons le $H$; on a $B=A \oplus H$. Supposons que $H$ soit un 
idéal fermé de $B$ et tel que $H^{2}=\{0\}$ ( $H$ idéal nilpotent de $B$ ). Si $x \in H$, l'idéal de $B$ engendré par $x$ est l'ensemble des $f(x)$ où $f$ est un polynôme du premier degré à coefficient dans $A$ et à terme constant nul; en effet, soit $A_{x}$ l'ensemble ainsi défini, $I_{x}$ l'idéal engendré par $x$; on a évidemment $A_{x} \subseteq I_{x}$.

Si $y \in I_{x}, y$ est une somme finie de produits finis de $R(x)$ où $R \in \mathscr{E}^{\prime}$ algèbre multiplicative de $B$. Or $R(x) R\left(x^{\prime}\right)=0$ car $H$ est nilpotent, donc les $R(x)$ sont des polynômes du premier degré en $x$. D'autre part, pour tout $m \in H$, pour tout $b \in J$ :

$$
R_{b+m}(x)=R_{b}(x) .
$$

Donc la restriction de $R_{b+m}$ à $H$ est la même que celle de $R_{b}$.

On peut donc considérer que $R \in \mathscr{E}$ algèbre multiplicative de $A$; alors $R \in A[X]$ et $\operatorname{deg} R=1$ donc $I_{x} \subseteq A_{x}$.

L'idéal engendré par $x$ dans $B$ est donc $A^{\prime}[x]$, ensemble des $f(x)$ où $f$ est un polynôme à terme constant nul et à coefficients dans $A$. On peut aussi écrire $I_{x}=\mathscr{E} x$. La sous-algèbre engendrée par $x$ est $\mathbb{C} x$, car $0 x$ $=x \cdot x=0.0=0$.

4. Corollaire. L'idéal fermé par $x \in H$ dans $B$ est $\mathscr{E} x$.

\section{Représentations cycliques}

1. Généralités et propriètés. Considérons maintenant une algèbre de Jordan normée complète, un espace de Hilbert $H, S$ une représentation cyclique de $J$ dans $H ; J \oplus H$ muni du produit $(x+m)(y+n)=x y+S_{y} m$ $+S_{x} n$ est une algèbre de Jordan. Comme $S$ est cyclique, on peut décomposer $H$ en $H_{1} \oplus H_{2}$ et $S$ en $U+V$ où $U$ et $V$ sont définis dans [4] et [5]. Ainsi: $J \oplus H=J \oplus H_{1} \oplus H_{2}$.

$J \oplus H_{1}$, est une sous-algèbre de $J \oplus H$ mais $J \oplus H_{1}$ n'a pas d'élément unité; $J \oplus \mathrm{H}_{2}$ est une sous-algèbre de $J \oplus H$ et $J \oplus H_{2} a$ un élément unité qui est celui de $J . J$ est une sous-algèbre unitaire de $J \oplus H_{2} . H_{2}$ est un idéal de $J \oplus H_{2}$ et $H_{2}^{2}=0$. $J$ est normée complète, il en est de même pour $\mathrm{H}_{2}$ donc $J \oplus H_{2}$ est normée, complète pour la norme:

$$
\|a+m\|=\sup _{\substack{a \in J \\ m \in H_{2}}}(\|a\|,\|m\|) .
$$

$J \oplus \mathrm{H}_{2}$ est donc une extension de $J$ et tout élément de $\mathrm{H}_{2}$ est algèbrique sur $J . J \oplus H_{2}$ est ainsi une extension algèbrique de $J$.

Soit $\xi_{2}$ le vecteur cyclique de $\mathrm{H}_{2}$. L'idéal engendré par $\xi_{2}$ dans $J \oplus \mathrm{H}_{2}$ est l'ensemble des $f\left(\xi_{2}\right)$ où $f$ est un polynôme de degré 1 à terme constant nul. Autrement dit c'est l'ensemble $\mathscr{E} \xi_{2}$ où $\mathscr{E}$ est l'algèbre multiplicative de $J$. La sous-algèbre de $J \oplus H_{2}$ engendrée par $J \cup\left\{\xi_{2}\right\}$ est l'ensemble des 
$f\left(\xi_{2}\right)$ où $f \in J[X]$, or si deg $f \geqq 2$ [8], on a $f\left(\xi_{2}\right)=0$ donc la sous-algèbre de $J \oplus H_{2}$ engendrée par $J \cup\left\{\xi_{2}\right\}$ est l'ensemble des $f\left(\xi_{2}\right)$ où $f \in J[X]$ est de degré 1 . L'extension simple de $J$ par adjonction de $\xi_{2}$ est donc égale à $J\left[\xi_{2}\right]=J \oplus \mathscr{E} \xi_{2}$.

Comme $S$ est cyclique, la fermeture de $J \oplus \mathscr{E} \xi_{2}$ est telle que: $\overline{J \oplus \mathscr{E} \xi_{2}}=J \oplus \overline{\mathscr{E} \xi_{2}}=J \oplus H_{2}$ donc $J \oplus \mathscr{E} \xi_{2}$ est partout dense dans $J \oplus H_{2}$.

Remarque. Par abus d'écriture on a noté $\mathscr{E}$ l'algèbre multiplicative de $J$. Montrons que si $\mathscr{E}$ désigne l'algèbre associative engendrée par les $S_{x}[4]$ et [5], $x \in J$, on a: $\mathscr{E} \xi_{2}=\mathscr{E}^{\prime} \xi_{2}$. En effet pour tout $x \operatorname{de} J, R_{x} \xi_{2}=S_{x} \xi_{2}$ $=V_{x} \xi_{2}$, donc les générateurs de $\mathscr{E} \xi_{2}$ et $\mathscr{E}^{\prime} \xi_{2}$ sont égaux donc $\mathscr{E} \xi_{2}=\mathscr{E}^{\prime} \xi_{2}$.

Signálons quelques propriètés relatives à la représentation régulière. Soit donc $R$ la représentation régulière de $J \oplus H_{2}$ on $a: R_{n+m}(x+y)$ $=x y+V_{x} n+V_{y} m$ et $R_{x} n=V_{x} n$, pour tout $n$ et pour tout $x$. Si dans $J \oplus H_{2}$, on a: $V_{x y}=\frac{1}{2}\left(V_{x} V_{y}+V_{y} V_{x}\right)$ alors pour tout $x, y \in J$, on a: $R_{x y} n=\frac{1}{2}\left(R_{x} R_{y}\right.$ $\left.+R_{y} R_{x}\right) n$ ce qui donne: $[x, y, n]=[n, x, y]=-[y, x, n]$ (associateurs dans le triplet de Lie de $J \oplus H_{2}$. De l'identité de Jacobi pour ces triplets de Lie on tire: $2[x, y, n]=[x, n, y]$, d'où $[x, y, n]=0 \Leftrightarrow[x, n, y]=0$.

Soit maintenant $I$ l'ensemble de $R \in \mathscr{E}$ tels que $R \xi_{2}=0$. C'est un idéal à gauche de $\mathscr{E}$ considérée comme une algèbre associative. $I$ est un idéal bilatère de l'algèbre de Jordan des polynômes sur $J . J \oplus \mathscr{E} \xi_{2}$ est isomorphe à $J[X] / I$.

2. Particularisation. Soit: $J$ algèbre de Jordan avec unité sur $\mathbb{C}, M$ espace vectoriel sur $\mathbb{C}, S$ représentation de $J$ sur $M$, telle que $S_{1}=1$ où 1 désigne à la fois l'unité de $J$ et l'opérateur identité sur $M$.

Considérons $J \oplus M$, c'est une algèbre de Jordan pour le produit défini précédemment; soit $x, y \in J, m, n \in M$. Considérons l'application $R$ de $J \oplus M$ dans l'ensemble des opérateurs linéaires de $J \oplus M$, telle que:

$R_{x}: a \rightarrow a x, \quad m \rightarrow S_{x} m=x m ; \quad R_{m}: a \rightarrow S_{a} m=a m, \quad n \rightarrow 0=m n$.

Comme $R_{x+m}=R_{x}+R_{m}$ alors $R_{x+m}(a+n)=(x+m)(a+n)$ donc $R$ est la représentation régulière de l'algèbre de Jordan $J \oplus M$.

Un élément unité de $J$ est aussi un élément de $J \oplus M$ car $1(x+m)$ $=1 x+1 m=x+S_{1} m=x+m$, d'où $R_{x+m} n=S_{x} n$, donc pour tout $m$, $R_{x+m}=S_{x}$.

L'étude de la représentation $S$ est ainsi ramenée à l'étude de la représentation régulière de $J \oplus M$. En regroupant les résultats précédents et en prenant les notations de (4) et (5), nous voyons que nous avons obtenu le théorème général ci-après.

3. Théorème. Toute R.G. S d'une algèbre de Jordan commutative, involutive, normée, complète, avec unité (notée: 1 ) telle que $S_{1} \neq 1$ (l'unité de l'algèbre de Jordan et l'opérateur unité sur l'espace de représentation sont toutes deux désignées par 1) peut se ramener à la somme directe de R.G. cycliques $S^{\prime}$ telles que $S_{1}^{\prime} \neq 1$ et chaque $S^{\prime}$ peut se décomposer à son

13 Commun. math. Phys., Vol. 16 
tour en la somme directe d'une R.S. cyclique $U$ et d'une R. $G_{1}$.V (telle que $\left.V_{1}=1\right)$ cyclique. Toute $R . G_{1}$. peut se ramener à une représentation régulière (R.R.) d'une algébre de Jordan convenablement choisie.

En conclusion, on a ramené toute étude d'une R. G. à celles d'une R.S. cyclique et d'une R.R.

Remerciements. Nous tenons à remercier Monsieur Störmer, pour les corrections qu'il a bien voulu porter à ce travail, ainsi que Monsieur le professeur D. Kastler pour son hospitalité à l'Institut de Physique Théorique de l'Université de Marseille.

\section{Bibliographie}

1. Jordan, P.: Commun. Math. Phys. 9, 279-292 (1968); 11, 293-296 (1959).

2. Ion Studii, B. P.: Cercetari Matematice 2, 17 (1965); 7, 18 (1966); 2, 19 (1967).

3. Gunsen, J.: Commun. Math. Phys. 6, 262-285 (1967).

4. Ravatin, J., Immédiato, H.: Compt. Rend. 266 A, 1019-1020 (1968).

5. - Math. Ann. (sous presse).

6. Brun, Koecher, M.: Jordan Algebren. Berlin-Heidelberg-New York: Springer 1966.

7. Bourbaki, N.: XI Algèbre. 1102. Paris: Herman 1965.

8. Ravatin, J., Immédiato, H.: Préprint. Faculté des Sciences de Lyon.

J. Ravatin

H. Immédiato

Faculté des Sciences

Institut de Mathématiques

43 Boul. du 11 Novembre 1918

F 69 Villeurbanne 